Cahiers d'études italiennes

\title{
Mésaventures de la religion-fiction : Roma senza papa de Guido Morselli
}

Pierre Laroche

\section{(2) OpenEdition}

\section{Journals}

Édition électronique

URL : http://journals.openedition.org/cei/224

DOI : $10.4000 /$ cei.224

ISSN : 2260-779X

Éditeur

UGA Éditions/Université Grenoble Alpes

Édition imprimée

Date de publication : 15 juillet 2009

Pagination : 297-306

ISBN : 978-2-84310-145-8

ISSN : 1770-9571

\section{Référence électronique}

Pierre Laroche, « Mésaventures de la religion-fiction : Roma senza papa de Guido Morselli », Cahiers d'études italiennes [En ligne], 9 | 2009, mis en ligne le 15 janvier 2011, consulté le 28 mars 2021. URL http://journals.openedition.org/cei/224 ; DOI : https://doi.org/10.4000/cei.224 


\title{
MÉSAVENTURES DE LA RELIGION-FICTION : ROMA SENZA PAPA DE GUIDO MORSELLI
}

\author{
Pierre Laroche \\ Université de la Sorbonne Nouvelle-Paris 3
}

Euvre posthume, comme tous les romans de Guido Morselli (I9I2-I973), publiée en 1986, Roma senza papa ${ }^{1}$, datée de 1966 par l'auteur, se situe à Rome en juin $199 .$. Senza papa, non qu'il n'y ait plus de pape, mais Jean XXIV, successeur de Paul VI et Libero ${ }^{\text {er }}$, a décidé de s'installer avec les institutions et organismes pontificaux à Zagarolo, à 36 kilomètres de Rome. Il y vit dans une résidence semblable à un ensemble de motels et s'y complaît dans la solitude et le silence. Personne ne sait pour quelles raisons ce bourg a été préféré à d'autres ( $R S P, \mathrm{p} . \mathrm{I} 52)$. Zagarolo, du $\mathrm{XI}^{\mathrm{e}}$ au $\mathrm{XVII}^{\mathrm{e}}$ siècle, et effet sous la domination de la famille Colonna; Pie IX lui a conféré le statut de città en I858. Quand Morselli écrivait Roma senza $p a p a$, cette ville comptait environ 6500 habitants (actuellement 13 000).

Morselli fait état du mécontentement des ecclésiastiques exilés dans ce bourg, de celui des Gardes suisses, armée dissoute de but en blanc, et des plaisanteries amères des Romains insatisfaits, en particulier du fait de la perte de revenus due à la chute de l'activité touristique de la Ville éternelle : le peuple romain se sent abandonné et les policiers sont inquiets pour leur emploi du fait qu'auparavant les voitures immatriculées S.C.V. représentaient, dit l'un d'eux au narrateur, Walter, la moitié de la circulation romaine. Ce narrateur relève des éléments de continuité et parle avec humour de la saleté : " $i$ locali [...] afosi e sporchetti circa come allora" (p. Iо), "la bambinocrazia [p. I7-19], [...] il calcio [...] motore della vita nazionale " (p. 52), le goût du bruit : "il bisogno [...] di sonorizzarsi "

I. Milano, Adelphi, 1986. Dans la suite de cet article : RSP.

2. Le gouvernement italien ayant décidé de réduire les émoluments des footballeurs professionnels, " $d a$ Ferrara a Siracusa, la piazza insorse. A Roma [...] fu proclamata la Repubblica autonoma della Lazio " (RSP, p. 58). 
(p. 6o). Pour lui, "questa città continua a fingere una delle possibili propedeutiche all'inferno " (p. I2). Walter est un prêtre suisse d'Einsiedeln, dans le canton de Schwytz, né en 1943, marié à Lotte qui considère qu'il est fidèle même dans ses rêves ; il est venu à Rome, parcourant à pied les trente derniers kilomètres, pour une audience du pape. L'audience se fait attendre plusieurs jours pendant lesquels il parcourt la Ville éternelle, où il a vécu trente ans auparavant, entre 1968 et 1972, et où il rencontre ou retrouve divers personnages, des ecclésiastiques pour la plupart, qui commentent de diverses façons les changements en cours dans le monde et surtout dans l'Église en cette fin fictionnelle du $\mathrm{xx}^{\mathrm{e}}$ siècle.

Ces discussions traitent des mutations de la société, des techniques, des mœurs, de la situation politique mondiale et surtout des changements en cours, espérés ou redoutés dans l'Église à la suite du II concile œcuménique de Vatican II $^{3}$, ouvert par Jean XXIII en 1962 et clos par Paul VI en 1965, un peu plus de trente ans avant le moment où est censé se situer le roman de Morselli, mais peu de temps avant sa rédaction. Ce concile, généralement caractérisé par l'ouverture du catholicisme au monde moderne, a traité des rapports de l'Église catholique avec les autres religions et avec la société contemporaine. Il intervenait dans une décennie marquée par des tentatives de libération politique, sociale et culturelle, en Afrique où la décolonisation ouvrait des espoirs de développement indépendant, en Europe occidentale et en Amérique du Nord où s'esquissait une remise en question des rapports de domination et d'exploitation, en Amérique du Sud surtout, pour ce qui concerne notre propos. La théologie de la libération, que l'on s'accorde à faire commencer en 1957 avec la création des communautés de base au Brésil, propose que la religion ait pour objet la liberté et la solidarité, contre l'aliénation économique et sociale. En I9841986, elle sera contestée par les travaux de la congrégation Propaganda Fide, engagés à la demande de Jean-Paul II, au prétexte que cette réflexion d'une partie importante des catholiques et du clergé d'Amérique du Sud liait la question de la rédemption à l'engagement politique et à la violence contre les dirigeants. Toutefois le pape Jean-Paul II, de même que le cardinal Ratzinger, reconnut dans les années 1990 que cette approche pouvait avoir des aspects positifs.

3. XXII ${ }^{e}$ concile œcuménique de l'Église catholique romaine, environ un siècle après Vatican I (I869-I870), lequel avait été convoqué par Pie IX - près de 300 ans après le précédent concile œcuménique de l'Église catholique romaine, le concile de Trente - et avait été marqué par les discussions sur les rapports entre la raison et la foi ainsi que sur la définition de l'infaillibilité pontificale, mais, interrompu par la guerre franco-prussienne, n'avait pu mener à leur terme les travaux portant sur l'Église elle-même. 
Roma senza papa porte donc sur des questions qui mettent en cause l'Église catholique dans son nouveau cours, dont le départ de Rome n'est après tout qu'un épiphénomène.

Morselli prend le risque de l'anticipation qui, à la différence de l'utopie, ne prétend pas imaginer les buts et les voies d'une société idéale, mais le monde tel qu'il deviendrait si les choses continuaient à évoluer de la façon dont, selon l'auteur, elles le font à l'heure où il écrit. Roma senza papa développe donc à sa manière les résultats des travaux de Vatican II. Cette extrapolation situe la fin du $\mathrm{xx}^{\mathrm{e}}$ siècle dans un monde où la haute technologie pénètre les rites, les sacrements, avec la création de confessionnaux automatiques, un monde où règnent des usages qui dépassent certaines revendications qui s'exprimeront en 1968 : marijuana en vente libre, hallucinogènes prescrits médicalement comme stimulants intellectuels - et la "Compagnia ", conformiste, condamne l'anticonformisme de ceux qui s'abstiennent d'en prendre (p. 27). Selon certains, les changements de l'Église catholique la rapprochent du protestantisme : elle se veut fondée sur la tolérance, vise l'œecuménisme "che tende a diminuire le differenze tra le varie accezioni cristiane» (p. 39) ; elle recherche le rapprochement avec le bouddhisme ; l'Église anglicane, mettant fin à un schisme de quatre siècles, a regagné l'Église catholique ; on intègre la psychanalyse dans la théologie et certains prêtres posent la question du mal de façon toute nouvelle, non comme résultat du péché originel, fruit du Malin, mais comme dû à l'inconscient ; l'athéisme est accepté comme foi, la foi est tenue pour une éthique et l'Église " ha mandato in pensione il Diavolo" (p. 157). En France et en Belgique, pour être ordonné prêtre, il faut un diplôme de métallurgiste. On dit la messe à Sélénopolis sur la Lune, et l'on procède à l'évangélisation des Vénusiens : on est loin de la polémique contre Galilée à propos de l'homme centre de la création ${ }^{4}$. Les jésuites instaurent en Italie du Sud une économie soviétiste efficace. Le mariage des prêtres est la norme. Sans parler du projet de pontificat à durée limitée ou, mais c'est à mettre au compte de l'humour du narrateur et de l'auteur, la pratique de certains prêtres, Américains abstèmes, qui remplacent le vin de messe par du Coca-Cola.

Cet humour tend à caricaturer la recherche de modernité de la part de l'Église qui cherche l'innovation avec une désinvolture acritique quand

4. Morselli revient sur cette question dans Dissipatio H. G. : "non esiste escatologia che non consideri la permanenza dell'uomo come essenziale alla permanenza delle cose. Si ammette che le cose possano cominciare prima, ma non che possano finire dopo di noi» (p. 56). 
l'un des interlocuteurs de Walter déclare (p. 35) : " ci deve essere una teologia dell'acculturazione, una teologia dell'automazione [...] Tante teologie quante sono le vie di Dio. Magari un po' meno, ma insomma..." Walter l'interrompt "con intenzione sarcastica. Anche una teologia sportiva. E un'altra, alimentare ", ou quand Morselli copie le langage de la publicité, créant un Centurvat (Centro turistico vaticano), donne une image de la carrière ecclésiastique qui repose largement sur l'arrivisme et la recommandation. Caricature assumée quand le narrateur reconnaît qu'il a évoqué sa carrière " col tono del carrierismo superficiale, da professionista della vocazione" (p. I3).

Inutile de poursuivre sur ce terrain : comme la science-fiction, la religion-fiction est démentie par la réalité ultérieure ; l'évolution historique ne se fait pas de façon mécanique en fonction de prétendues tendances lourdes, elle inclut des imprévus et des impondérables, l'action ou l'inaction des individus et des collectifs, des forces et des faits économiques, sociaux, politiques, culturels, idéologiques, moraux. Traitant des besoins de programmation en matière d'emploi et de formation universitaire, le sociologue François Dubet écrit "que les anticipations [...] restent très incertaines " (in " le Snesup ", mai 2006, p. I5). Que dire alors de l'entreprise de Morselli qui porte sur une projection concernant un ensemble culturel, idéologique et moral de l'envergure d'une des principales religions dites monothéistes ? On peut constater par exemple que Morselli n'imagine pas dans sa fiction que ce qui marquera dans le réel la période où il la situe n'est pas l'évolution de la religion catholique, mais les rapports entre les religions, entre les religions et les sociétés, les religions et les États, dans un contexte où ce que certains appellent «l'empire - ou l'axe du mal » n'est plus le même. Les rapports entre chrétienté et islam, entre christianisme, islam et religion juive, entre politique et religion, n'ont pas dans ce roman la place qu'ils ont prise dans la politique mondiale après sa rédaction (la guerre des Six Jours intervient un an après la date de 1966 donnée par Morselli pour l'écriture de son roman). Si Morselli a déjà une idée de ce qui est devenu la mondialisation, il ne fait aucune place aux problèmes environnementaux et n'entrevoit pas plus que tous ses contemporains la disparition des systèmes politiques d'Europe de l'" Est ", alors qu'on vient de passer à l'ère Brejnev après la période où Khrouchtchev dirigeait l'Union soviétique. Et le narrateur affirme sa certitude mathématique que, en l'an 2000, le PCI accèdera légalement au pouvoir en Italie alors qu'il s'est auto-dissout en I99I. Et Morselli ne prévoyait certainement pas que, en 1998, dans la période où il situe Roma senza papa, un ministre des Finances allemand Theodor Waigel, parlerait, à propos de l'Italie et 
des pays de l'Europe du Sud, de «pays du club Méd ». Et pourtant, « edificata l'Europa", écrit Morselli, "gli Italiani si sentono tutti indistintamente retrocessi a "Sud" [...]. Ci hanno detto [...] che le nostre fabbriche di auto, di frigoriferi erano relitti di un passato autarchico. "Da voi, solo il sole" " (RSP, p. II).

Il ne s'agit donc pas de découvrir que Morselli s'est trompé dans cette tentative de religion-fiction. Comme tout bon humoriste, il doit être pris au sérieux, mais pas pris au mot et il ne croit certainement pas au futur qu'il dessine. Il ne raconte pas une allégorie ou un apologue. À peine une histoire. En supposant pour la fin du $\mathrm{xx}^{\mathrm{e}}$ siècle des évolutions de la religion qui se poursuivraient dans le sens qu'il croit ou prétend voir aux évolutions liées à Vatican II et aux questions qui se posent à l'Église, sur elle-même, sur les rapports sociaux, la sexualité, la mort, Morselli est amené à des réflexions qui sont de l'ordre du rationnel mais portent sur des domaines qui ne sont pas de cet ordre. Ces réflexions sous-entendent un jugement sur les évolutions de l'Église comme sur celles de la société où elle intervient, mais Morselli exprime, sans doute plus ou moins volontairement, des incertitudes sur ces points.

Le pape de son roman, Jean XXIV, est un Irlandais entre deux âges, fiancé à une théosophe de Bangalore. Notre prêtre suisse trouve quelque chose d'oriental à ce pape qui a été délégué apostolique en Irak où il a fréquenté le patriarche des nestoriens ${ }^{5}$ et l'un de ses objectifs est d'arriver à résoudre les différences entre ces deux Églises pour ramener les nestoriens, puis l'Église orthodoxe, "alla comune fonte cristiana" (p. I56-I57) ${ }^{6}$. En effet, si une motivation du déménagement du siège pontifical est le souci d'alléger les procédures - «se durava troppa fatica a anna' dal Papa. Doveva esse' più semplice, più alla portata " dit un Garde suisse mis à la retraite (p. 54-55). La préoccupation de Jean XXIV est qu'en restant " romain ", le pape ne peut être "catholique " au plein sens du terme; le transfert à Zagarolo et l'hypothèse d'une mobilité constante, y compris hors d'Italie,

5. Église syro-araméenne, issue du conflit du v siècle (au centre notamment du Concile de Chalcédoine en 45I) entre dyophysites et monophysites, portant sur la question de savoir si le Christ a deux natures, une divine et une humaine, ou une seule nature qui réunit ces deux composantes. Nestorius trancha le débat en faisant de Marie, non la mère de Dieu ou la mère de l'homme, mais la mère du Christ. L'Église nestorienne en tire comme conséquence le refus de la Trinité et se place hors de l'Église de Rome, tout en gardant le titre de catholicos pour le patriarche de l'Église arménienne. Les schismes n'étaient pas réglés au sein de cette Église au moment où Morselli rédigeait Roma senza papa: ce n'est qu'en 1995 que le catholicos de Cilicie, installé à Beyrouth, a été élu patriarche suprême et catholicos de tous les Arméniens. Morselli se réferre-t-il à cette " querelle byzantine " sur la nature du Christ quand il qualifie la Vierge de "theotokos, madre di suo figlio " ? (RST, p. I43)

6. Est-ce une référence à la rencontre de Paul VI, lors de son premier voyage à l'étranger, à Jérusalem, en janvier 1964, avec le patriarche œcuménique de Constantinople (Église orthodoxe de Constantinople), Athénagoras $\mathrm{I}^{\mathrm{er}}$ ? 
jusqu'à la grotte de Bethléem (p. I54), entre donc dans sa conception du rôle universel de l'Église ${ }^{7}$. On apprend bientôt qu'il vient d'obtenir la responsabilité mondiale de la répartition des zones d'influence, y compris sur la Lune, entre les États-Unis et l'Union soviétique, et la fin du roman fait de cette Église catholique inattendue "una terza potenza a vantare dei diritti» (p. I83). On pourrait certes dire que Jean-Paul II a réellement fait, ou refait, de l'Église une puissance qui a pesé sur l'évolution politique du monde, mais sans rien à voir avec le roman de Morselli, lequel n'explique rien de ce qui permet à l'Église de Jean XXIV de devenir cette " troisième puissance " : son pragmatisme, sa conception œcuménique de la religion ? On ne sait pas par quelles voies, théologiques, culturelles, idéologiques, politiques, économiques cette démarche a pu progresser. Walter le pressent quand il tente de définir les raisons qui ont présidé au choix du nom de Jean XXIV : après deux papes, Paul VI et Libero ${ }^{\text {er }}$, qui ont choisi « una santità e bontà di accesso indiretto", plus intellectuelle en quelque sorte, il revient à la "bontà un po' facile " de Jean XXIII (p. I76).

Walter, très traditionaliste, continue à revêtir les habits sacerdotaux, à commencer la messe par l'Introibo, et voit en Pie XII "l'ultimo papa che sia stato a tutti gli effetti regnante" (p. 24). Il se sent témoin, mais non participant, de la fin d'une période historique. La ville suisse d'où il vient est connue pour son abbaye du XvinI ${ }^{\mathrm{e}}$ siècle mais, plus encore, pour son Carnaval dont les masques sont des diables et Walter confirme qu'il continue de croire au Diable. Il voit aussi dans le «bric-à-brac » des miracles, non "la superstizione [...] ma la pretta, l'antica, la venerabile cattolicità del senso e del sentimento, irriflessa e dunque diretta, autentica" (p. IOo) ; Morselli le met d'ailleurs en contradiction avec les faits le jour où il voit défiler un cortège de cardinaux et de gardes suisses sur la Place Saint-Pierre, cortège qui disparaît soudain parce qu'il s'agit d'une sorte d'hologramme virtuel : est-ce la technique humaine qui accomplit les miracles? Walter est surtout l'auteur d'une Difesa dell'Iperdulia ${ }^{8}$, dont il attend - en vain, il le sait - que la revue Civiltà cattolica donne " una mezza colonna di recensione ". Il veut, grâce à son ouvrage, "vedere l'ortodossia prendere posizione di fronte all'eresia. Non si può continuare con $i$

7. Et non pas le souci de réduire les frais du Vatican, ni celui de s'éloigner des communistes de la mairie de Rome (" ils y sont depuis si longtemps ", dit Walter, qui, comme Morselli, n’y a pourtant vu ni Giulio Carlo Argan, ni Luigi Petroselli, ni Ugo Vetere, maires communistes de Rome dans les années 1970-1980) ou de la proximité du Général des Jésuites : il Papa nero n'a pas besoin d'être proche : il fait deux fois par jour le trajet Rome-New York et retour (RSP, p. I53-I54).

8. Hyperdulie : culte rendu à la Vierge, à la différence de "dulie ", culte rendu aux saints, et de "latrie ", culte rendu à Dieu. 
polls e le interviste a discutere se la Madonna sia un'ebrea convertita come tante altre, o nella migliore ipotesi una santa» (p. 15 ). De façon générale, il conteste plus ou moins explicitement non seulement les abandons de l'Église dans les domaines pastoral et doctrinal, mais le fait que ces questions deviennent "argomento di pubblica opinione » (p. I5), débattu à la télévision, par des laïcs : il n'accepte pas "la volgarizzazione o laicizzazione della sfera ecclesiastica e religiosa" (p. I6). Pour lui, conformément aux conceptions conservatrices d'une Église conçue non pas comme assemblée des fidèles mais fondée sur la séparation entre le clergé et le peuple, la religion s'adresse à tout un chacun, mais la théologie, le dogme, le rite, " una fondamentale tradizione, e istituzione", sont l'affaire de " $i$ legittimi, e cioè $i$ competenti ", parmi lesquels il se range, lui, "il modesto teologo d'Einsiedeln" (p. I6) où le contact entre il et modesto a tout d'un oxymore. Il n'est cependant pas le seul à constater et juger les évolutions de l'Église. Et quand deux jeunes prêtres exposent la "socialidarietà ", le doute s'insinue en lui : il reconnaît in petto qu'il est attiré par cette proposition qui, dit-on, sera l'un des principaux champs de bataille du Concile Latran VI, "il Concilio del Duemila ", mais on perçoit dans ses hésitations les échos des débats sur la théologie de la libération. Walter commente : "quando si è capito che tutti i paesi erano solidali di fatto [...] allora gli aiuti sono cominciati sul serio" (p. 7I). Mais il constate aussi que la charité est perdante dans cette mutation qui s'inspire non de l'Évangile mais du "gretto istinto della mutualità, hodie tibi, cras mihi [...]. La psicoanalisi, novant'anni fa, ha liquidato quel poco che restava dell'esorcistica". La socialidarietà, feint de s'interroger l'un des tenants de ce projet, "farà lo stesso con quello che rimane della carità cristiana?" .

On peut alors soupçonner Walter d'être un peu le porte-parole de Morselli. Les argumentations de celui-ci, contrairement à ce qu'il a dit, répété et écrit dans ses carnets ${ }^{9}$ sur le rôle prédominant de l'idéologie, laissent parfois penser qu'il croyait quand même à une place centrale de l'économie dans le malheur du monde. Dans Dissipatio H. G. (pour humani generis $^{\mathrm{I}}{ }^{\mathrm{O}}$, il parle de la ville de Crisopoli, ville de la richesse comme son nom l'indique, "le sue radici attingono l'æternum del capitale, quintessenza della realtà » (p. 36-37). La plus grave faute des hommes a été «se non di cominciare la Storia, di proseguirla ». Les fautes les plus graves qu'il reproche à l'histoire des hommes ne sont pas d'ordre idéologique, mais

9. Cf. la thèse de D. Vittoz, La quête d'identité d'un intellectuel italien entre fascisme et après-fascisme : écriture d'essai et écriture de fiction chez Guido Morselli (1912-1973), 2 vol., Grenoble, 1995, p. 567.

Io. Milano, Adelphi, I977. 
ce sont "l'Imbruttimento, l'Inferocimento (con eufemismo, la "violenza"). L'inflazione (senza eufemismo: la peste monetaria)" (p. 65). Il s'agit alors non d'une question théologique mais philosophique et politique : si la mondialisation n'est pas une relation entre égaux, ce n'est pas qu'on n'ait pas compris que "la crisi dell'acciaio inglese fa pagare più tasse ai contribuenti americani " (p 69) ou que, si " un linfogranuloma [viene] curato e guarito in un ospedale di Tokio, è una notizia che reca sollievo a Lei a Roma, a me in Svizzera» (p. 73), mais qu'il y a peut-être des intérêts économiques qui motiveraient des résistances politiques et idéologiques à la socialidarité. Mais Walter juge que, désormais, structure et superstructure ne se distinguent plus : "quello che ha i piedi nell'economia ha il capo o il busto intero nella morale. O nella religione» (p. 70), faisant de l'économie, par cette métaphore, ce que certains appellent la base, et de la morale et la religion des superstructures.

Que Walter - et donc Morselli - soit réticent voire critique devant certaines tendances nouvelles de l'Église et prenne parfois des positions pour le moins conservatrices, on peut en avoir la confirmation chez le protagoniste de Dissipatio, réfractaire même à la doctrine sociale de l'Église, telle qu'elle remonte à l'encyclique Rerum Novarum (I89I) qui voyait dans le travail et non dans le capital, "la source féconde " de tous les biens, précisant que « le travail a une telle fécondité et une telle efficacité, que l'on peut affirmer sans crainte de se tromper que, seul, il donne aux nations la prospérité », ce qui met en porte-à-faux l'affirmation de Morselli selon laquelle le capital serait " la quintessence de la réalité ». Walter relève bien d'autres contradictions entre les positions traditionnelles et celles qu'exprime récemment l'Église : comment Paul VI pouvait-il dire que la paix est le bien suprême? "Una volta, ben supremo era la fede [...] anche a costo della guerra" (p. 86). Ses hésitations et ses doutes, comme sans doute les inquiétudes de Morselli, ne touchent pas que le domaine pastoral, le rôle du Pape et de l'Église catholique sur le monde, mais le domaine doctrinal, proprement théologique : même s'il reprend des débats vieux comme le christianisme ou peut-être même plus, Roma senza papa aborde vraiment, avec sérieux, des questions qui touchent au dogme, sous l'angle des raisons de la foi, dans ses rapports avec la raison, bien que les religions exigent toujours un sacrifice de l'intellect : la nature de Dieu (est-il bon, est-il juste, est-il tout-puissant, raisonnable, compréhensible ?), qui a le droit de le juger dans ses contradictions, quels rapports entre Dieu et l'homme, entre toute-puissance divine et libre arbitre de l'homme, quelle est l'origine du mal ? Sur toutes ces questions, il relève ou laisse apparaître des contradictions ou l'absence de réponses. 
La question du mal est, sans doute pour des raisons autobiographiques, la guerre notamment, l'objet d'une réflexion récurrente chez Morselli au plan philosophique. Ici, c'est de l'origine du mal qu'il traite, d'un point de vue théologique, en liaison encore avec son expérience personnelle : cette question occupe notamment plusieurs pages sous couvert d'un débat entre deux machines qui sont censées discuter de la virginité de Marie, mais l'un de ces engins (appelons-les " ordinateurs »), devenu incontrôlable, sort du sujet et pose explicitement la grande question : Unde malum? omniprésente dans le roman (p. 48-49, 9I-92, I43-I46). Adam, créature parfaite d'un auteur parfait, ne pouvait concevoir le mal. Si on déplace la responsabilité sur le serpent, le Diable, on n'a rien résolu : Dieu ne peut avoir créé le Diable. Ou alors, il est absurde de dire "délivre-nous du mal »si, comme le dit Isaïe, c'est Dieu qui a voulu et créé le mal (p. 9I). Et " un creatore in ipotesi perfetto, può forse commettere ingiustizie nella creazione?" (p. I43) : pourquoi alors des papillons ont-ils des tumeurs et pourquoi les châtaigniers sont-ils "scomparsi dalla faccia della Terra, distrutti da un morbo inarrestabile che chiamiamo fitocancro" (p. I46) ? En expiation de quoi et avec quel espoir de rédemption ? Ils n'ont quand même pas commis le péché originel dont les hommes ne se sont rendus coupables qu'après la création des végétaux et des animaux (p. I45).

Le regard de Morselli sur les évolutions de la société, et surtout sa virulence dans le rappel de questions et contradictions qui surgissent du christianisme ne supposent donc pas une acceptation de ce qui est. Il hésite entre contestation du catholicisme traditionnel et critique des changements en germe qu'il traite - de façon parfois un peu sommaire - par la dérision, sans doute parce qu'il les trouve dérisoires. L'humour de Walter ne porte pas que sur les évolutions de l'Église et de la religion, mais aussi sur lui-même, ses doutes, sur les autres religions, sur l'humanité. À travers le regard ironique qu'il porte sur les Italiens, les Suisses, les prêtres de toutes nationalités, il y a sans doute l'amertume devant la vanité de toute chose et de tout geste, la déception existentielle qu'il a explicitement déclarée dans ses carnets ${ }^{\text {II }}$. Une déception qui a peut-être parmi ses causes les constantes déceptions que lui ont valu ses œuvres littéraires, et dont on peut parfois lire la trace dans le roman ${ }^{12}$.

Au dernier chapitre de Roma senza papa, Walter est finalement reçu, dans un groupe de douze prêtres, par le Pape qui leur délivre un message

II. La thèse de D. Vittoz, op. cit., débute par ce constat (p. 2).

I2. Par exemple, le protagoniste de Dissipatio H. G. est soumis aux clauses léonines des contrats des éditeurs (p. 89). 
sibyllin : contestant implicitement la phrase de la Genèse (I : 27) selon laquelle "Dieu créa l'homme à son image ", Jean XXIV déclare : "Invece bisogna persuadersi che Dio è diverso, Dio non è prete [...] e nemmeno frate " (p. I76-I77). Cette affirmation de la différence absolue de Dieu laisse tous ces prêtres désorientés ; Walter en est troublé au point de chanceler physiquement. Jean XXIV met une limite à la fonction pastorale du Pape et de l'Église, s'en remettant ainsi totalement à la Grâce. Walter ne peut plus que se soustraire à ce qu'il appelle son "fluttuare interno, più che mai insidioso in questo estremo di crisi che il mondo spirituale attraversa, in questa incertezza di ogni credente creatura" (p. I8I).

Le pessimisme de Morselli, enraciné dans son expérience et dans sa réflexion, ne se satisfait pas de l'humanité, de son passé ni de son présent et le futur ne lui dit rien qui vaille : quand il réécrit les suites de la Première guerre mondiale (Contropassato prossimo), quand il feint d'écrire l'histoire à venir (Roma senza papa), quand il écrit, pour sa dernière œuvre, la disparition de l'humanité (Dissipation H. G.), en abolissant, non la vie, mais seulement les hommes, on peut penser que, pour lui, les refus systématiques, ou qui peuvent paraitre tels, de la part des éditeurs, ne sont qu'une métaphore de ce que l'humanité a de décevant, cette humanité qu'il qualifie de "banale ": Walter juge son attachement à Rome "profano, banale oltretutto. Umano" (RSP, p. 9) et le narrateur de Dissipatio se demandant s'il ne vit pas un rêve, commente "la domanda è banale e umana come uno sbadiglio" (p. 57). L'humanité est sans recours. L'histoire est sans recours (p. 26). Même la religion a déçu dans le passé et s'annonce comme décevante dans le futur; elle ne vaut pas de vivre et ne permet pas de vivre : le pape, avec ou sans Rome, n'offre pas de perspective ${ }^{13}$. En ce sens, sans qu'on veuille y trouver une dimension prémonitoire, les doutes et réticences de Morselli dans ce roman sont probablement indicatifs des doutes et réticences qui ont accueilli Vatican II et qui éclairent certaines évolutions de l'Église depuis Jean-Paul II.

13. Les réserves formulées par Walter sur Paul VI portent sur un pape non fictif dont Morselli a vécu une bonne partie du pontificat (1963-1978). 